\section{Real Personalized Medicine}

\author{
James O. Armitage, MD; William C. Wood, MD; and Dan L. Longo, MD
}

Being a physician is complicated. After all, we are not fixing a broken car or other machine; we are trying to help another human being. In the treatment of patients with a curable malignancy, the need to balance maximizing cure rates against minimizing seriousand sometimes lethal toxicities that often occur late in treatment is increasingly common. This balance is usually less of an issue in diseases in which cure is infrequent and survival is short. In this situation, trying to maximize cure rate is the overriding factor. However, in cancers in which the chance for cure is very high, particularly if second-line therapy also presents a possibility of cure, the situation is much more problematic.

Examples of the difficult decisions that physicians and patients must address can be found in of early-stage Hodgkin lymphoma (HL) and of ductal carcinoma in situ (DCIS) of the breast. HL was a uniformly fatal disease until reports on the use of radiotherapy by early investigators, including Peters and Middlemiss, ${ }^{1}$ Kaplan, ${ }^{2}$ and Tubiana et $\mathrm{al}^{3}$ showed that some patients with relatively limited disease appeared to be cured. However, follow-up of these patients showed serious long-term toxicities associated with radiotherapy as it was then administered. 4,5 Although median survival was extended with radiotherapy, most patients with HL eventually died of the disease. It was not until the combination chemotherapy regimen MOPP (mechloethamine, vincristine, procarbazine, and prednisolone) was used that experts noted a significant reduction in the number of deaths from HL. ${ }^{6}$ Subsequent studies showed that (1) adding an effective chemotherapy regimen to radiotherapy improved the cure rate, (2) the results seemed to be better with chemotherapy followed by radiotherapy in patients at high risk, and, more recently, (3) the use of chemotherapy alone was effective in patients with disease of all stages.

The ABVD (doxorubicin, bleomycin, vinblastine, dacarbazine) regimen generally does not cause infertility and has a small risk of secondary neoplasms. Investigators have shown equivalent survival in patients with earlystage, low-risk HL who experience a complete remission with a brief course of ABVD alone and with the same brief course of ABVD followed by involvedfield radiotherapy. ${ }^{7}$ The chances of remaining continuously disease-free for 3 years was higher for patients who received radiotherapy (95\% vs $91 \%$ with ABVD alone), with equivalent overall survival ( $97 \%$ vs $99 \%$, respectively). The overall survival might eventually alter in favor of the group that received radiotherapy as a result of eventual death from lymphoma for some of the patients who experienced relapse, or may show an advantage for no radiotherapy because of the eventual impact of second cancers and vascular disease related to the radiation therapy. However, although the overall survival seems to be similar, a slightly higher relapse rate consistently seems to occur when radiotherapy is not administered.,

Thus, the physician and patient are left balancing the advantage of no relapse (ie, avoiding psychologic distress, a potential bone marrow transplant, and the possibility of a slight decrease in survival) versus the benefits of avoiding radiotherapy (ie, reduced risk of a second cancer, reduced risk of cardiovascular disease, avoiding the acute toxicities of radiotherapy, and reduced cost of thera-

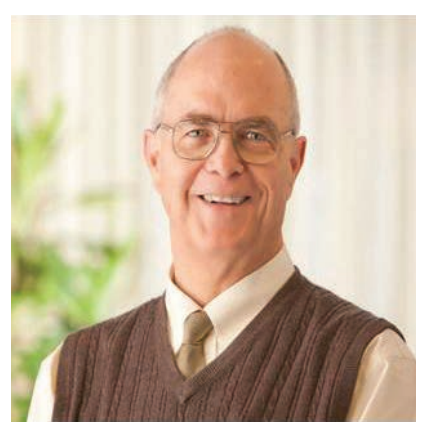

James O. Armitage, MD

Dr. James $\mathrm{O}$. Armitage is currently the Joe Shapiro Professor of Medicine at the University of Nebraska Medical Center. He is board certified in internal medicine, medical oncology, and hematology, and a Fellow of both the American and Royal College of Physicians and of the American Association for the Advancement of Sciences. He has served on many national/international oncology committees, including the United States National Cancer Advisory Board and the French National Cancer Advisory Board. He is past president of both ASCO and the American Society of Blood and Marrow Transplantation and has served on multiple international committees. He has numerous honors, including the Claude Jacquillat Award for achievement in Clinical Oncology, the San Salvatore Foundation Research Award, and the Richard and Hinda Rosenthal Foundation Award from the American Association of Cancer Research. Dr. Armitage has published more than 562 papers and written 110 chapters, and is co-editor of 27 books.

The ideas and viewpoints expressed in this commentary are those of the author and do not necessarily represent any policy, position, or program of $\mathrm{NCCN}$. 


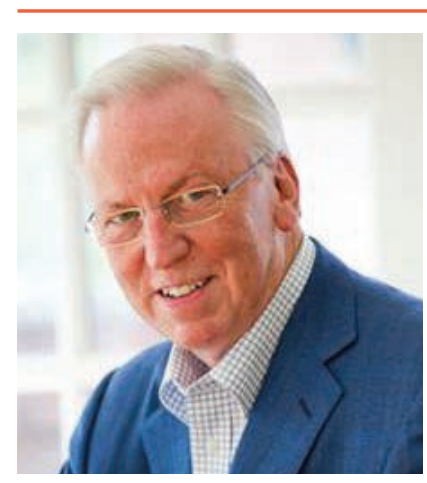

William C. Wood, MD

William C. Wood, MD, is Distinguished Joseph Brown Whitehead Professor of Surgery at Emory University School of Medicine and Professor of Global Health in the Hubert Department of Global Health in the Rollins School of Public Health. Since 2012 he has served as Academic Dean of PAACS' ten surgical residency programs in Africa. He has devoted his research efforts to oncology, first in the immunology of autoantibodies to tumor associated antigens, then in clinical trials of multimodality treatment of breast cancer. He is a Senior Research Fellow of the International Prevention Research Institute of Lyon, France, and serves on the Board of Advisors of the Africa Oxford Cancer Foundation, Oxford. He has served as Chair of the Cancer and Leukemia Group B Breast Committee, Chair of the Eastern Cooperative Oncology Group Breast Committee, Chair of the $\mathrm{NCl}$ Breast Intergroup, Co-Chair of the Early Breast Cancer Trialists' Collaborative Group (Oxford), Co-Chair of the NCl Breast Cancer Steering Committee, and $\mathrm{Co}-\mathrm{Ch}$ air of BIG-NABCG. He has been a Governor of the American College of Surgeons, a Director of ASCO, a member of the Board of Scientific Advisors of $\mathrm{NCl}$, and President of the Society of Surgical Oncology, the Southeastern Surgical Congress, the Georgia Surgical Association, the Atlanta Surgical Association, and the Southern Surgical Association. He is Editorin-Chief of Oncology, Associate Editor of Annals of Surgical Oncology, and has written over 280 full-length scientific articles, over 35 book chapters, and has edited or co-edited 7 books, including the 3-volume Oxford Textbook of Surgery, 2nd Ed. py). Modern radiotherapy techniques seem to have reduced the long-term risks associated with radiation, but those risks remain greater than with no radiation.

A similar situation can be found in decisions regarding the management of DCIS treated with surgical excision. Patients treated with surgery alone have a local recurrence rate of $28 \%$ at 10 years, but adjuvant radiation therapy lowers the rate to $13 \% .{ }^{9}$ No evidence of a survival difference is seen. Making the decision more fraught, $30 \%$ of women receiving adjuvant breast irradiation in good centers rate their cosmetic effect at fair to poor by 3 years. ${ }^{10}$ Any recurrence in that population leads to a recommendation of mastectomy, but patients who did not undergo radiation therapy are candidates for breastconserving treatment should their disease recur.

No randomized trials comparing mastectomy with breast-conserving surgery plus radiation therapy in DCIS are available. Several studies compare local excision to local excision plus whole-breast radiation (with or without tamoxifen), ${ }^{9}$ and all showed improved local control with the addition of whole-breast radiation therapy. However, no studies were powered to assess the effect on survival. So the question to patients becomes, are you optimistic that you will be in the group that does not experience a recurrence? Or would you rather not live with the constant threat that recurrence or progression to invasive disease may occur? The decision becomes an emotional one because no data are available to guide it. We can't discern which patients will experience recurrence or progression and which will not.

In both diseases, the addition of radiation therapy to lower the recurrence rate will result in many patients receiving treatment with only a few receiving benefit from that treatment. What are you more afraid of: recurrence or the treatment to prevent recurrence? Like Clint Eastwood's character Harry Callahan said, you have to ask yourself, "do you feel lucky?"

Studies that compare treatments-and that would assist these decisionscan look for superiority (ie, that one treatment clearly has a better outcome), or for noninferiority (ie, that within preset statistical boundaries, it cannot be shown that one treatment is worse than another). The study by Radford et $\mathrm{al}^{7}$ notes the complexities involved in interpreting this information to make a treatment decision. Their study of early-stage HL comparing a brief course of ABVD versus a brief course of ABVD followed by radiotherapy in patients in remission was designed as a noninferiority study. However, the authors originally planned that if the patients receiving chemotherapy had a durable remission rate not more than $10 \%$ lower than those receiving chemotherapy and radiotherapy (ie, a difference that might not have been expected to translate in improved overall survival), the study would demonstrate noninferiority. However, after the study was started, the investigators decided to reduce the target for noninferiority to $7 \%$.

As it turned out, the study showed that the 95\% confidence limit was between $1.3 \%$ better and $8 \%$ worse progression-free survival for the chemotherapy alone arm. If the original 10\% margin had been kept, this study would have shown noninferiority, but because of the change to $7 \%$, the study did not meet the noninferiority margin. But which limit was correct: the first decision (in which case the 2 regimens would be considered noninferior to each other) or the second decision (that the progression-free survival of the chemotherapy alone arm missed the target for noninferiority)? 
Will the advent of precision medicine solve these dilemmas? Treatment choices such as these would not be so complicated if we had biomarkers to identify which patients with early-stage HL would be cured with ABVD alone and which would require radiotherapy. Because approximately $90 \%$ of patients with HL are cured with chemotherapy alone, we are actually looking for a way to identify the $4 \%$ or so of patients who require additional therapy. Similarly, if we knew which cases of DCIS would not relapse after local excision alone, determining which patients need radiotherapy would be easy.

Examples of cases in which we have this information are increasing. We have known for decades that patients whose breast cancers express estrogen receptors are likely to respond to hormonal manipulations, whereas patients whose tumors do not express hormone receptors will not benefit. Patients with acute lymphoblastic leukemia whose cancer has the Philadelphia chromosome and expresses BCR-ABL obtain great benefit from imatinib and similar drugs, but other patients with acute lymphoblastic leukemia do not benefit. ${ }^{11,12} \mathrm{Re}$ cently, we have seen that patients with non-small cell lung cancer (NSCLC) whose cancers have epidermal growth factor receptor rearrangements are likely to benefit from drugs such as erlotinib, ${ }^{13}$ and those with anaplastic lymphoma kinase rearrangements have a high likelihood of benefiting from crizotinib, ${ }^{14}$ but most patients with NSCLC benefit from neither drug.

Unfortunately, to date no equivalent markers are available to identify which patients with early-stage HL or DCIS can be safely treated with, respectively, a brief course of chemotherapy or local excision. In the case of HL, negative PET scan results after a brief course of chemotherapy indicate patients for whom observation might be reasonable, but even these patients show a slightly higher relapse rate if radiotherapy is not administered.?

In the case of DCIS of the breast, unifocality, smaller lesion size, and age older than 50 years are all signs of less risk of recurrence. Available genomic risk scores provide a low-risk indication for $60 \%$ to $70 \%$ of such women in addition to the standard clinicopathologic factors.

Who should make the decision, the physician? Presumably, the physician can be objective and is the most knowledgeable about the data. However, physicians also have potential conflicts of interest related to treatment decisions. Patients certainly have the right to make the decision. After all, it's their body. However, patients are more likely to be subject to optimistic bias, such as the mistaken belief that one's chances of experiencing a perceived negative event are lower than for other similar patients. Finally, the decision could be made by society. As health care costs increase, pressure to make treatments available based on cost-effectiveness is increasing. Of course, this requires assigning values to certain outcomes and determining the dollar amount of the outcomes.

In general, we believe that this should be the patient's decision, made in concert with an interested, caring, and informed physician. Not every patient will make the same decision. For some, the risk of relapse will be so terrifying that further therapy to minimize the risk of relapse will be desirable, despite attendant adverse events. Other patients will most fear the possibility of a second cancer or increased risk of coronary artery disease, or just fear undergoing radiotherapy. These patients will accept the already high chance of doing well with observation as the best option.

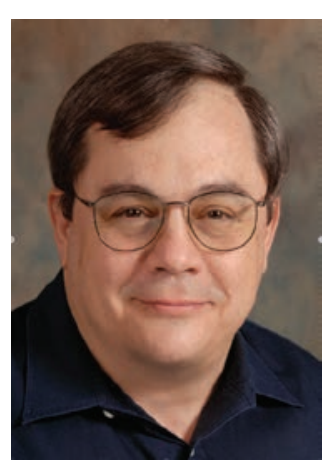

Dan L. Longo, MD

Dr. Dan L. Longo is Professor of Medicine at Harvard Medical School in the Division of Hematology at Brigham and Women's Hospital and Deputy Editor of the New England Journal of Medicine. He is a Master of the American College of Physicians, a member of the American Society of Clinical Investigation and the Association of American Physicians, and a fellow of the American Association for the Advancement of Science. He has been active in research on the treatment of malignant lymphoma, including new treatments targeting CD30, CD40, HLA Class II molecules, and immunoglobulin idiotype. His laboratory work has focused in part on the regulation of lymphocyte proliferation, natural killer cell effects on hematopoiesis, and tumorinduced immunosuppression. He has published over 900 peerreviewed papers, review articles, editorials, and book chapters and has been an editor of Harrison's Principles of Internal Medicine since 1994, including serving as Editor-in-Chief of the 18th edition. He has written or edited 25 books. 
Armitage et al

If ever effective communication between physicians and patients is need$\mathrm{ed}$, it is during this decision-making process. This is certainly not a time to be looking at the computer rather than at the patient. This is also not something best done in a 12- to 15-minute office visit. The complexity of risk factors and the lack of proven survival benefit from adjuvant radiation are sufficient to demand an informed, compassionate physician who has time to listen to the patient's concerns and values. Communication is also not likely to be effective without a trusting and respectful relationship already in place. This is the sort of "personalized medicine" that effective physicians have been practicing since before the term became part of our medical vernacular.

\section{References}

1. Peters MV, Middlemiss KC. A study of Hodgkin's disease treated by irradiation. Am J Roentgenol Radium Ther Nucl Med 1958;79:114-121.

2. Kaplan HS. The radical radiotherapy of regionally localized Hodgkin's disease. Radiology $1962 ; 78: 553-561$.

3. Tubiana M, Rambert P, Laugier A, Lalanne CM. Extended irradiation in Hodgkin's disease: early reactions. Nouv Rev Fr Hematol 1966;6:164-174.

4. $\mathrm{Ng} \mathrm{AK}$, Bernardo P, Weller E, et al. Patients with early-stage Hodgkin's disease treated at age 50 or younger. J Clin Oncol 2002;20:2101-2108.

5. Swerdlow AJ, Higgins CD, Smith P, et al. Myocardial infarction mortality risk after treatment for Hodgkin disease: a collaborative British cohort study. J Natl Cancer Inst 2007;99:206-214.

6. Canellos GP, Rosenberg SA, Friedberg JW, et al. Treatment of Hodgkin lymphoma: a 50-year perspective. J Clin Oncol 2014;32:163-168.

7. Radford J, Illidge T, Counsell N, et al. Results of a trial of PET-directed therapy for early-stage Hodgkin's lymphoma. N Engl J Med 2015;372:1598-1607.

8. Raemaekers JM, Andre MP, Federico M, et al. Omitting radiotherapy in early positron emission tomographynegative stage I/II Hodgkin lymphoma is associated with an increased risk of early relapse: clinical results of the preplanned interim analysis of the randomized EORTC/LYSA/FIL H10 trial. J Clin Oncol 2014;32:1188-1194.

9. Early Breast Cancer Trialists' Collaborative Group. Overview of the randomized trials of radiotherapy in ductal carcinoma in situ of the breast. J Natl Cancer Inst Monogr 2010;2010:162-177.

10. Eblan MJ, Vanderwalde NA, Zeman EM, Jones E. Hypofractionation for breast cancer: lessons learned from our neighbors to the north and across the pond. Oncology 2014;28:536-546.

11. Thomas DA, Faderi S, Cortes J, et al. Treatment of Philadelphia chromosome-positive acute lymphocytic leukemia with hyper-CVAD and imatinib mesylate. Blood 2004;103:4396-4407.

12. De Labarthe A, Rousselot $P$, Huguet-Rigal F, et al. Imatinib combined with induction or consolidation chemotherapy in patients with de novo Philadelphia chromosome-positive acute lymphoblastic leukemia: results of the GRAAPH-2003 study. Blood 2007;109:1408-1413.

13. Mok TS, Wu YL, Thongprasert S, et al. Fefitinib or carboplatin-paclitaxel in pulmonary adenocarcinoma. N Engl J Med 2009;361:947-957.

14. Kwak EL, Bang YJ, Camidge R, et al. Anaplastic lymphoma kinase inhibition in non-small cell lung cancer. $\mathrm{N}$ Engl J Med 2010;363:1693-1703. 